\title{
Seasonal incidence, correlation and regression among weather parameters against mites on summer okra
}

\author{
Y.T. JADHAV*, S.R. MANE ${ }^{1}$ AND D.S. SHINDE ${ }^{2}$ \\ Department of Agricultural Entomology, Ratnai Agriculture College, AKLUJ (M.S.) INDIA \\ ${ }^{1}$ Department of Horticulture, Ratnai Agriculture College, AKLUJ (M.S.) INDIA \\ ${ }^{2}$ Department of Agricultural Entomology, College of Agriculture, PANIV (M.S.) INDIA
}

\section{ARITCLE INFO}

Received : 14.06 .2016

Revised : 24.08 .2016

Accepted : 08.09.2016

\section{KEY WORDS :}

Linear, Multiple, Regression, Constant, Significant

*Corresponding author: Email : rupayogeshjadhav@gmail.com

\begin{abstract}
On summer okra crop seasonal incidence as influenced by weather parameters on mites population reached its peak during last week of April with 8.40 mites in $6.25 \mathrm{~cm}^{2}$ leaf area/ 3 leaves. The correlation between mite population was positively significant against maximum temperature $\left(\mathrm{r}=0.841^{* *}\right)$, minimum temperature $\left(\mathrm{r}=0.805^{* *}\right)$, evaporation $\left(\mathrm{r}=0.803^{* *}\right)$, wind velocity $\left(\mathrm{r}=0.728^{* *}\right)$ and bright sunshine hours $(\mathrm{r}=$ $\left.0.649^{*}\right)$, while with morning R.H $\left(\mathrm{r}=-0.717^{* *}\right)$ and evening R.H $\left(\mathrm{r}=-0.643^{*}\right)$ it was negatively significant. The equations of linear and multiple regression were set of mite population by working out regression co-efficient (b) and constant (a) alongwith coefficient of determination $\left(\mathrm{R}^{2}\right)$.
\end{abstract}

How to view point the article : Jadhav, Y.T., Mane, S.R. and Shinde, D.S. (2016). Seasonal incidence, correlation and regression among weather parameters against mites on summer okra. Internat. J. Plant Protec., 9(2) : 494-497, DOI : 10.15740/HAS/IJPP/9.2/494-497. 